\title{
NUMERICAL MODELING OF FOUNDATION SLAB WITH CONCENTRATED LOAD
}

\author{
${ }^{1}$ Josef FIEDLER, ${ }^{2}$ Tomáš KOUDELKA \\ Department of Mechanics, Faculty of Civil Engineering \\ Czech Technical University in Prague, Thákurova 7/2077, 16629 Prague, Czech Republic \\ e-mail: ${ }^{1}$ josef.fiedler@fsv.cvut.cz, ${ }^{2}$ koudelka@cml.fsv.cvut.cz
}

Received 30 December 2015; accepted 21 June 2016

\begin{abstract}
Several nonlinear simulations of concrete and reinforced concrete slabs are performed using a layered model. Two Drucker-Prager criteria are employed to form a concrete plasticity model that is used for simulating the plastic yielding of layers. Moreover, an interaction with elastic Winkler-Pasternak subsoil model is considered for the case of a reinforced concrete foundation slab that is subjected to a concentrated loading force. All computations are done by the SIFEL solver using finite element method.
\end{abstract}

Keywords: Foundation slab, Layered model, Double Drucker-Prager criterion, Concrete plasticity model, Finite element method

\section{Introduction}

Concrete foundation slabs are common problem in engineering world and, in general, the designing process does not cause many difficulties. But in certain cases, especially for industrial buildings, there are specific requirements and boundary conditions that may certainly transform the designing process into a challenging task. In general, there are two main areas of interest. The first one is connected with the determination of load-bearing capacity while taking into account crack development in concrete material [1]. The other is then consisted of using advanced materials, e.g. fiber concrete, instead of reinforced concrete and examining the effect of fiber density on load-bearing capacity [2]. In many of these situations a nonlinear calculation has to be applied. A foundation slab can be modeled by the full 3D model where, except of the suitable model for subsoil, no additional treatments are required. In other approach, the 
slab can be represented by plate elements with two parametric subsoil enhancements. For this purpose, the layered model [3] needs to be employed so the material parameters across the thickness of the slab can be included. The number of unknowns is, compared to the 3D model, significantly reduced and the variability of the 3D model is preserved because different material models can be applied to each layer.

The SIFEL software package [4], [5] has been extended by a new material class representing the layered model. Also, for better approximation of the nonlinear behavior, concrete plasticity model using two Drucker-Prager criteria has been added. With the help of the software and the new models, the simulation of a reinforced concrete foundation slab has been performed and the simulation was motivated by a real engineering problem of the heavy-loaded foundation slab in a storage hall. SIFEL has already been used for solving various nonlinear calculations such as [6], [7], [8].

The paper is mainly focused on the implementation of the layered model into the SIFEL software, while further information about the concrete plasticity model is possible to find in [9]. The first part of the paper is dedicated to the basic description of the layered model and the following part to the principles of the implementation. Then, few examples of basic slabs simulation are shown and finally the analysis of the foundation slab including interaction with subsoil is examined.

\section{Description of layered model}

The deformation of a general shell structure in $x, y$ plane can be described by the middle plane strains $\boldsymbol{\varepsilon}_{0}$ and the curvatures $\boldsymbol{\kappa}$ as follows

$$
\boldsymbol{\varepsilon}_{0}=\left[\varepsilon_{0, x}, \varepsilon_{0, y}, \gamma_{0, x y}\right]^{T}, \quad \boldsymbol{\kappa}=\left[\kappa_{x}, \kappa_{y}, \kappa_{x y}\right]^{T}
$$

By the layered model, this structure can be divided into small layers where each layer is considered to be in the plane stress state defined by following strain and stress components

$$
\boldsymbol{\varepsilon}=\left[\varepsilon_{x}, \varepsilon_{y}, \gamma_{x y}\right]^{T}, \quad \boldsymbol{\sigma}=\left[\sigma_{x}, \sigma_{y}, \tau_{x y}\right]^{T}
$$

The strain components of the $j$-th layer can be evaluated as

$$
\boldsymbol{\varepsilon}_{j}=\boldsymbol{\varepsilon}_{0}+z_{j} \mathbf{\kappa}
$$

where $z_{j}$ is the distance of the $j$-th layer from the middle of the plane, depicted in Fig. 1 .

From the strains, the stress components can be computed

$$
\boldsymbol{\sigma}_{j}=\mathbf{D}_{j} \boldsymbol{\varepsilon}_{j}
$$


where $\mathbf{D}_{j}$ is considered to be the stiffness matrix for the plane stress state of the $j$-th layer. The final product of the layered model is the determination of the stress resultant forces

$$
\mathbf{n}=\left[n_{x}, n_{y}, n_{x y}\right]^{T}, \mathbf{m}=\left[m_{x}, m_{y}, m_{x y}\right]^{T},
$$

that are computed as the summation of the contributions from all layers. The single contribution of the $j$-th layer is calculated by the following expressions

$$
\boldsymbol{n}_{j}=\boldsymbol{t}_{j} \boldsymbol{\sigma}_{j}, \quad \boldsymbol{m}_{j}=z_{j} \boldsymbol{t}_{j} \boldsymbol{\sigma}_{j},
$$

where $\mathbf{t}_{j}$ stands for the thickness of the current layer. After combining (3)-(6), the relation between the strains of the structure and the stress resultant forces can be obtained

$$
\begin{aligned}
& {\left[\begin{array}{l}
\mathbf{n} \\
\mathbf{m}
\end{array}\right]=\sum_{j}\left[\begin{array}{cc}
\mathbf{t}_{j} \mathbf{D}_{j} & z_{j} \mathbf{t}_{j} \mathbf{D}_{j} \\
z_{j} \mathbf{t}_{j} \mathbf{D}_{j} & z_{j}^{2} \mathbf{t}_{j} \mathbf{D}_{j}
\end{array}\right]\left[\begin{array}{l}
\boldsymbol{\varepsilon} \\
\mathbf{\kappa}
\end{array}\right] .}
\end{aligned}
$$

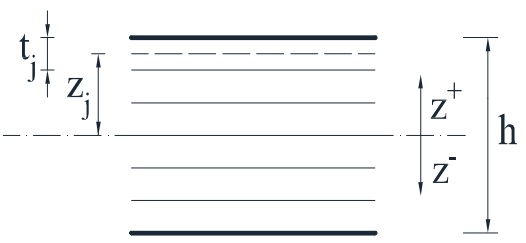
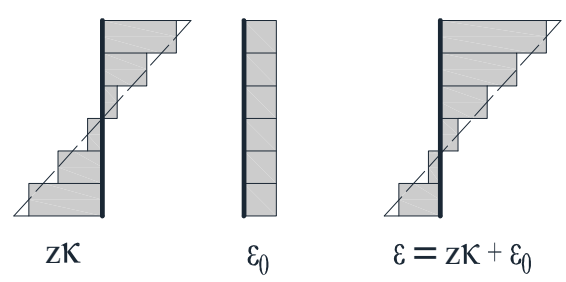

Fig. 1. Decomposition of a structure into layers

\section{Modification of layered model for plates}

In the calculation using plate elements, the only unknowns are the curvatures $\boldsymbol{\kappa}$ and there is only the moment stress resultant force $\mathbf{m}$. This is derived from the linear theory of plates where the neutral plane is considered to be identified with the middle plane and the normal component of the stress resultant forces is equal to zero. When applying the layered model on plates, the assumptions of the linear theory of plates have to be reconsidered. Since each layer can be assigned various stiffness matrixes, the neutral plane does not have to coincide with the middle plane. The main intention of the modification is to establish the stiffness matrix that appears in the following expression

$$
\mathbf{m}=\mathbf{D}_{e, s t i f f} \mathbf{\kappa},
$$


while considering the possibility of non-uniform distribution of layer stiffness.

Starting with an assumption that $\mathbf{n}=[\mathbf{0}]$, the system equation of (7) can be rewritten into the form

$$
\left[\begin{array}{l}
\mathbf{0} \\
\mathbf{m}
\end{array}\right]=\sum_{j}\left[\begin{array}{ll}
\mathbf{D}_{N N} & \mathbf{D}_{N M} \\
\mathbf{D}_{M N} & \mathbf{D}_{M M}
\end{array}\right]\left[\begin{array}{l}
\boldsymbol{\varepsilon}_{0} \\
\mathbf{\kappa}
\end{array}\right],
$$

where

$$
\begin{aligned}
& \mathbf{D}_{N N}=\sum_{j} \mathbf{t}_{j} \mathbf{D}_{j}, \\
& \mathbf{D}_{N M}=\mathbf{D}_{M N}=\sum_{j} z_{j} \mathbf{t}_{j} \mathbf{D}_{j}, \\
& \mathbf{D}_{M M}=\sum_{j} z_{j}^{2} \mathbf{t}_{j} \mathbf{D}_{j} .
\end{aligned}
$$

From the first row of (9), the vector of the middle plane strain can be derived

$$
\boldsymbol{\varepsilon}_{0}=-\mathbf{D}_{N N}^{-1} \mathbf{D}_{N M} \mathbf{\kappa}
$$

After substituting (11) into the second row of (9), the relation between the moment components and the curvatures including the requested stiffness matrix can be obtained

$$
\mathbf{m}=\left(-\mathbf{D}_{M N} \mathbf{D}_{N N}^{-1} \mathbf{D}_{N M}+\mathbf{D}_{M M}\right) \mathbf{\kappa}=\mathbf{D}_{e, s t i f f} \mathbf{\kappa}
$$

\section{Implementation and nonlinear behavior of layers}

The implementation is based on the idea that layers can be assigned with various material models. In principle, there is a separate calculation on each layer that determines the stress components, based on the computed strain components of a layer, using the prescribed material model. Using this approach allows to perform full nonlinear calculation of a cross-section. But, considering nonlinear behavior of layers almost every time leads to an imbalance of the normal components of the stress resultant forces and that is, with respect to the plate theory, inadmissible. Therefore, a correcting procedure needs to be applied so the balance of the normal components is sustained during the whole calculation.

The procedure consists of an iterative algorithm that, on the basis of the imbalance of the normal components, calculates an additional middle plane strain and searches for new position of the neutral plane. In the first iteration step, both normal $\mathbf{n}^{i}$ and moment $\mathbf{m}^{i}$ components of the stress resultant forces are evaluated. The Euclidean norm of the 
residual $\mathbf{n}^{i}$ is then compared with the required accuracy err (the superscript $i$ denotes the inner iteration step)

$$
\text { if }\left\|\mathbf{n}^{i}\right\|_{2}<e r r \text {. }
$$

If the condition in (13) is satisfied the procedure is terminated, otherwise the position of the neutral plane needs to be corrected and the additional middle plane strain is calculated by the following expression

$$
\Delta \boldsymbol{\varepsilon}_{0}^{i}=\mathbf{D}_{N N}^{-1} \mathbf{n}^{i}
$$

The new additional middle plane strains are then added to the total strains

$$
\varepsilon_{0}^{i+1}=\varepsilon_{0}^{i}+\Delta \varepsilon_{0}^{i}
$$

that proceed to the next step $(i+1)$ where the new stress resultant forces are computed and again compared with (13). This iteration process continues until the normal component equilibrium is reached.

\section{Computation example}

In this chapter, a simple example of a rectangular slab is presented. There are two sets of results in which the computational effect of the layered model should be demonstrated. The rectangular slab $(1.0 \mathrm{~m} / 1.65 \mathrm{~m})$ is formed of DKT plate elements [10] (the topology is shown in Fig. 2) and is supported simply alongside the upper and the side edges while the lower edge is fixed. The cross-section of the slab is $100 \mathrm{~mm}$ thick and, for the first set of results, divided into 10 layers all of them assigned with concrete plasticity model using two Drucker-Prager criteria (plain concrete) [11]. In the case of the second sets of results, two reinforcement layers with $J_{2}$ plasticity model [11] are added to the both surfaces of the slab (reinforced concrete). The following parameters are used for concrete: compressive strength $f_{c}=10 \mathrm{MPa}$, tensile strength $f_{t}=$ $1 \mathrm{MPa}$ and the Young's modulus $E=30 \mathrm{GPa}$. The reinforcement layers are given the following values: $A_{s}=500 \mathrm{~mm}^{2} / \mathrm{m}$, yield strength $f_{y}=500 \mathrm{MPa}$ and the Young's modulus $E=200 \mathrm{GPa}$. The slab is loaded uniformly and the initial value of the load is $0.2 \mathrm{kN} . \mathrm{m}^{2}$. During the calculation, the load is increased in particular load steps by virtue of gradually increasing load factor $\lambda$. While using DKT elements, it should be also noted that calculation is based on the Kirchhoff theory of plates therefore the effect of shear deformation is not considered.

The load factor $\lambda$, in the case of plain concrete, has reached the value of 220 and the results are presented in Fig. 3 - Fig. 5. In Fig. 3, the plastic multiplier $\gamma$, that represents the magnitude of plastic strains in the current area, is shown. As expected, 
the major plastic strains appear alongside the fixed edge and there are also the minor plastic strains in the sagging moment area of the slab.

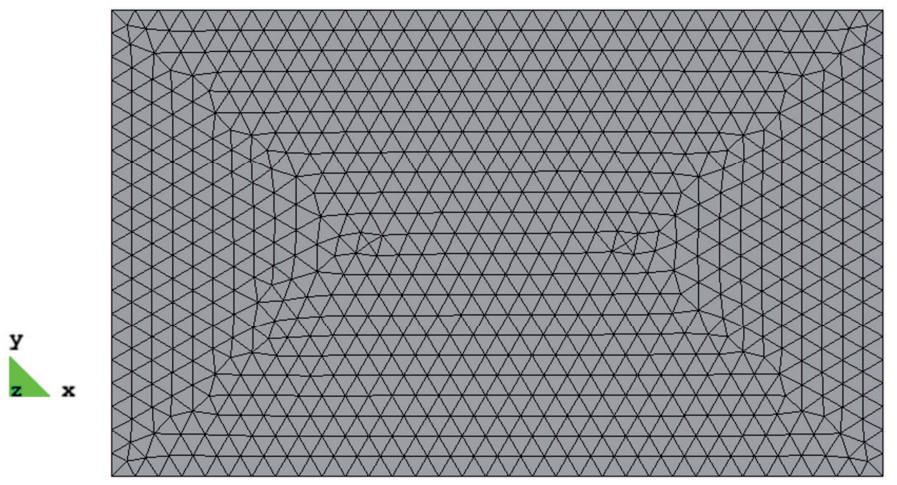

Fig. 2. Topology of the rectangular slab

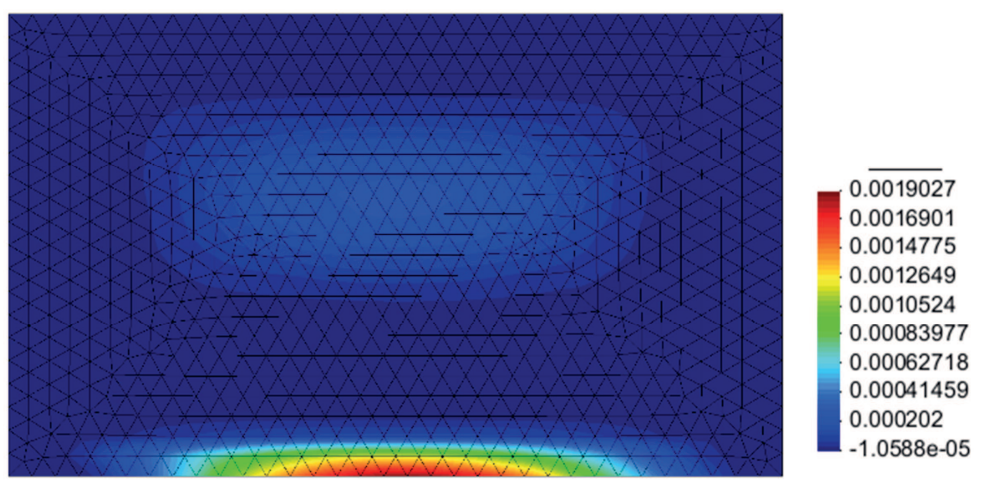

Fig. 3. Cumulative plastic multiplier $\gamma[-]$

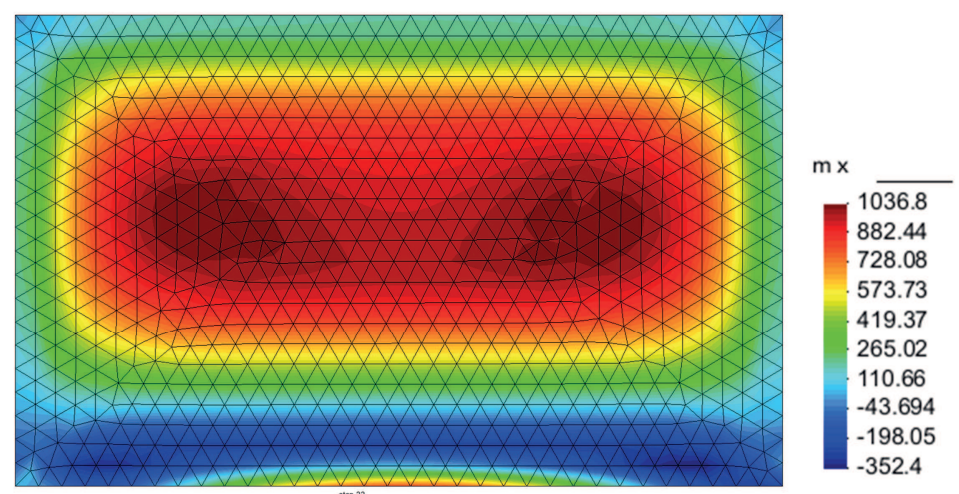

Fig. 4. Bending moment $m_{x}[\mathrm{Nm}]$

Pollack Periodica 11, 2016, 3 


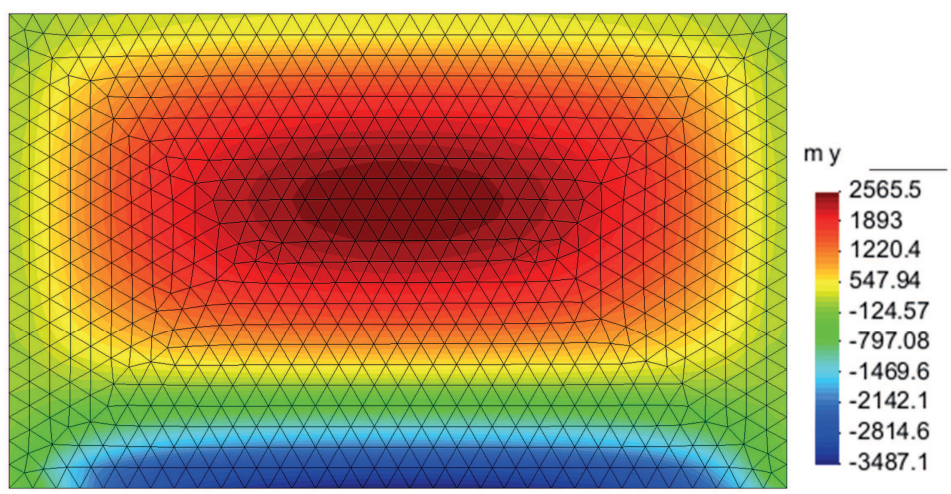

Fig. 5. Bending moment $m_{y}[\mathrm{Nm}]$

In the case of reinforced concrete, the load factor has reached approximately the value of 1400 . The effect of adding the reinforcement layers can be clearly observed on every variable presented in Fig. 6 - Fig. 8.
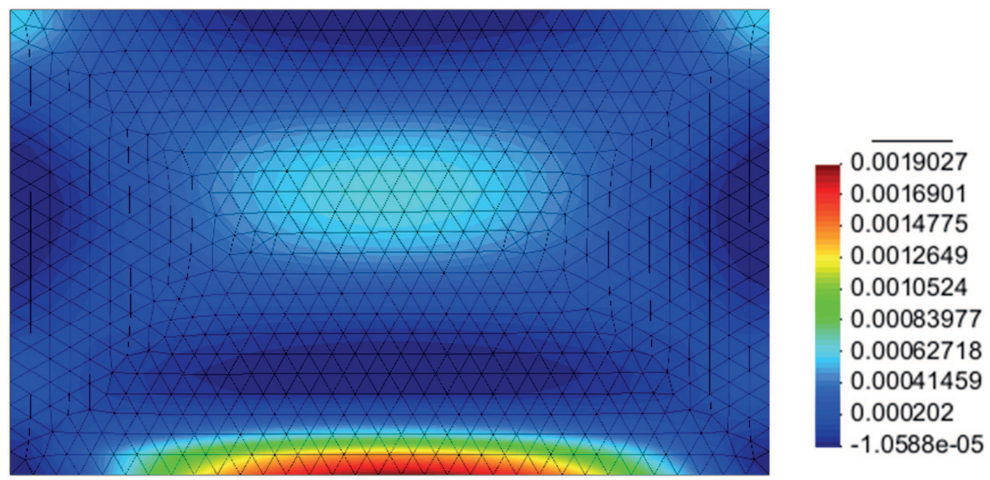

Fig. 6. Cumulative plastic multiplier $\gamma[-]$

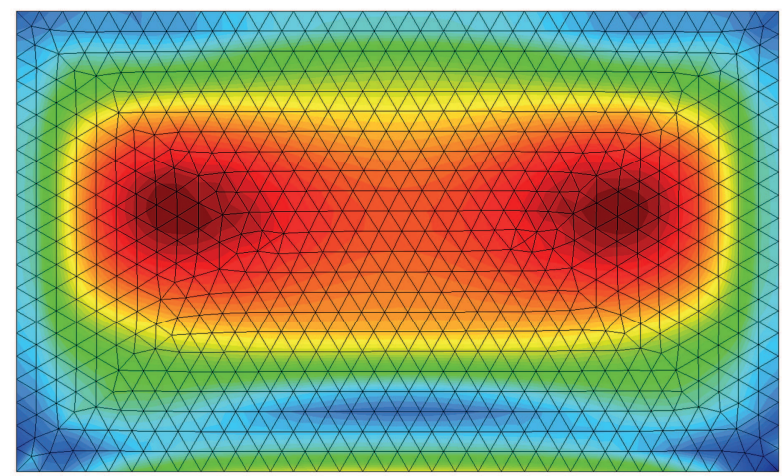

$\mathrm{mx}$
3412.1
2939.5
2467
1994.4
1521.8
1049.3
576.71
104.14
$-368.42$
$-840.99$

Fig. 7. Bending moment $m_{x}[\mathrm{Nm}]$ 


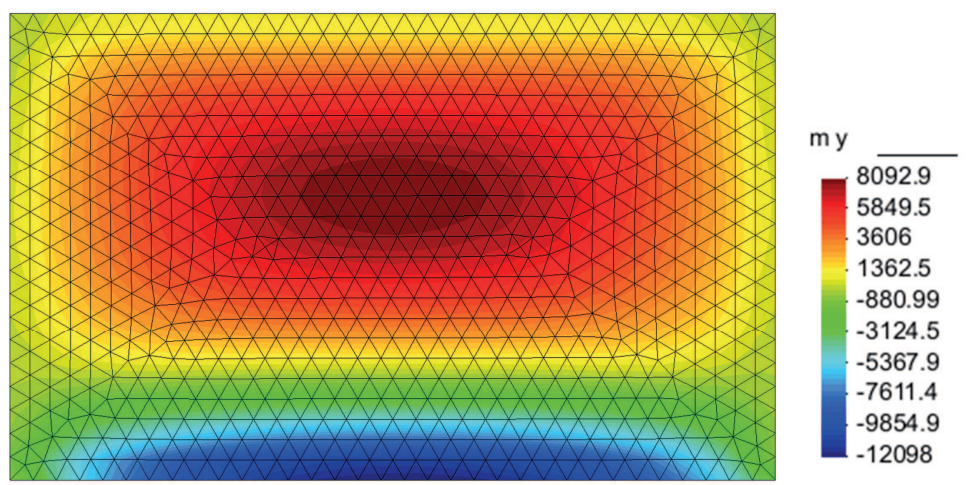

Fig. 8. Bending moment $m_{y}[\mathrm{Nm}]$

The major difference can be then clearly recognized in the distribution of the plastic multiplier (Fig. 6 ) where a different cracking mechanism of the slab can be observed.

\section{Foundation slab}

The described implementation was motivated by a real engineering problem of a foundation slab in a storage hall. This slab was placed between two compressors intended to push natural gas into a depository beneath the ground level. The idea was to design a slab that is able to resist a concentrated load in the form of a mobile crane. The following analysis is meant to verify the existing design and to establish the actual load capacity of the foundation slab. The layered model is applied mainly to include nonlinear behavior and the effect of reinforcement. For the detailed modeling of interaction between reinforcement and concrete, it is also possible to use more advanced approach that is based, for example, on the FETI method [12].

The topology is depicted in Fig. 9 where the green part represents the DKT plate elements of the slab and the grey elements, which are also implemented in the area of the slab, simulate the behavior of surrounding subsoil using two-parametric elastic soil model.

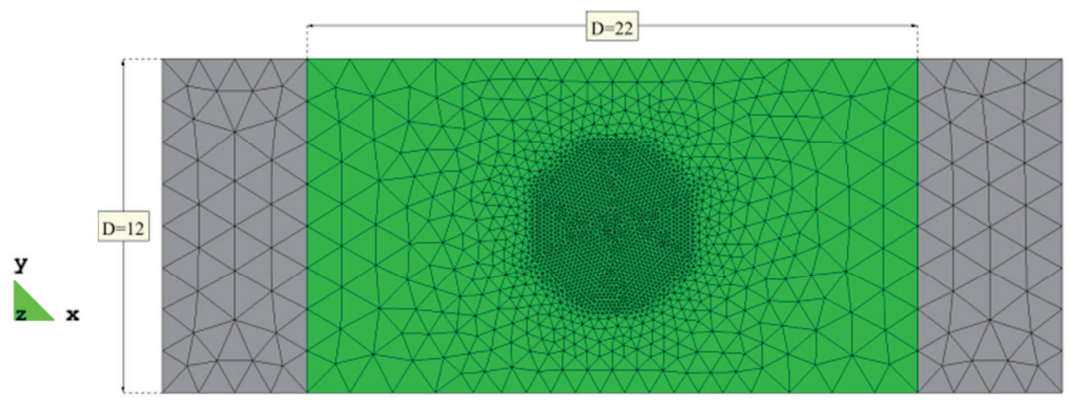

Fig. 9. Topology of the area $[\mathrm{m}]$ 
The cross-section of the slab is modeled by ten layers of concrete (20 mm thick each) applied with the double Drucker-Prager model and two reinforcement layers $(0.5 \mathrm{~mm}$ thick each - corresponding with $A_{s}=500 \mathrm{~mm}^{2} / \mathrm{m}^{\prime}$ ) with the $J_{2}$ plasticity model (Fig. 10a). The loading scheme representing a mobile crane is presented in Fig. $10 \mathrm{~b}$ and it is applied into the middle of the foundation slab where the mesh density has been increased due to the expectation of plastic strain development.

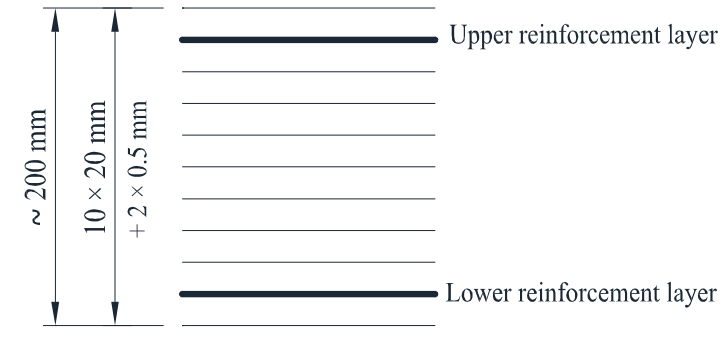

a)

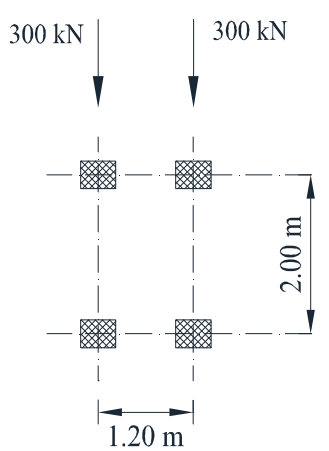

b)

Fig. 10. a) Layered model of the foundation slab, b) loading scheme of a mobile crane

The following parameters are used for the analysis: $f_{c}=30 \mathrm{MPa}, f_{t}=3 \mathrm{MPa}$, $E_{c}=30 \mathrm{GPa}, A_{s}=500 \mathrm{~mm}^{2} / \mathrm{m}^{\prime}, f_{y}=500 \mathrm{MPa}, E_{s}=210 \mathrm{GPa}$. The soil model is set by parameters $C_{1}=3.0 \mathrm{MPa} \cdot \mathrm{m}^{-1}, C_{2}=1.0 \mathrm{MPa} . \mathrm{m}$ and the initial value of the load is set to $1 / 30$ of the loading scheme. The nonlinear calculation, due to large amount of output data, has been stopped when the 5.8 multiple of the loading scheme has been attained. The results are displayed in Fig. 11 - Fig. 13.

It stems from Fig. 11 and Fig. 12 that the major bending moments are located right beneath the location of the load. As expected, the major plastic strains (Fig. 13) refer to this maximum bending moment area. While these positive moments are important for the determination of the load bearing capacity, there are also the negative moments (the dark blue area in Fig. 11 and Fig. 12) that are decisive from the serviceability point of view because they can be the source of cracks at the visible upper surface of the slab. In the presented case where the load factor has reached the value of 5.8, the circular light blue area can be observed in Fig. 13. If compared to the location of the negative moments, the development of the plastic strains indicates that cracks at the visible surface of the slab are very likely, in this case, to occur.

\section{Conclusion}

The paper has been mainly focused on the description of the layered model and its specific use for plate structures. Furthermore, the implementation of the model into the SIFEL software has been presented by using modifications for nonlinear behavior of layers. At the end, the effect of the layered model has been demonstrated on the simple 
rectangular slab subjected to plasticity calculation followed by the nonlinear analysis of the reinforced concrete foundation slab. From the analysis, it has been observed that the foundation slab is capable of resisting the load represented by the 5.8 multiple of a mobile crane but not without cracks development at the visible surface of the slab.

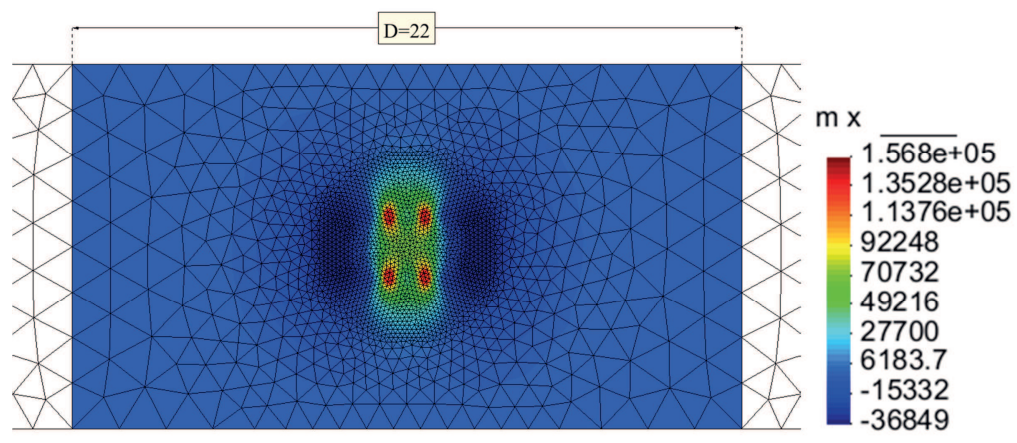

Fig. 11. Bending moment $m_{x}[\mathrm{Nm}]$

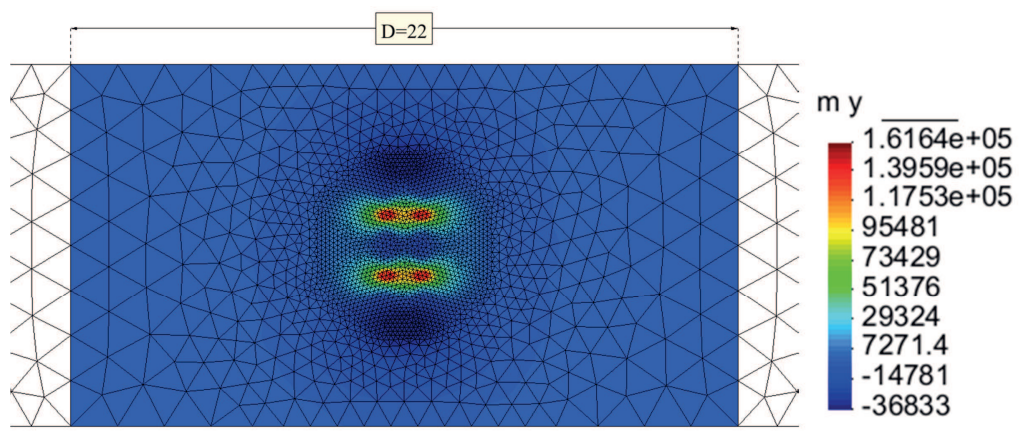

Fig. 12. Bending moment $m_{y}[\mathrm{Nm}]$

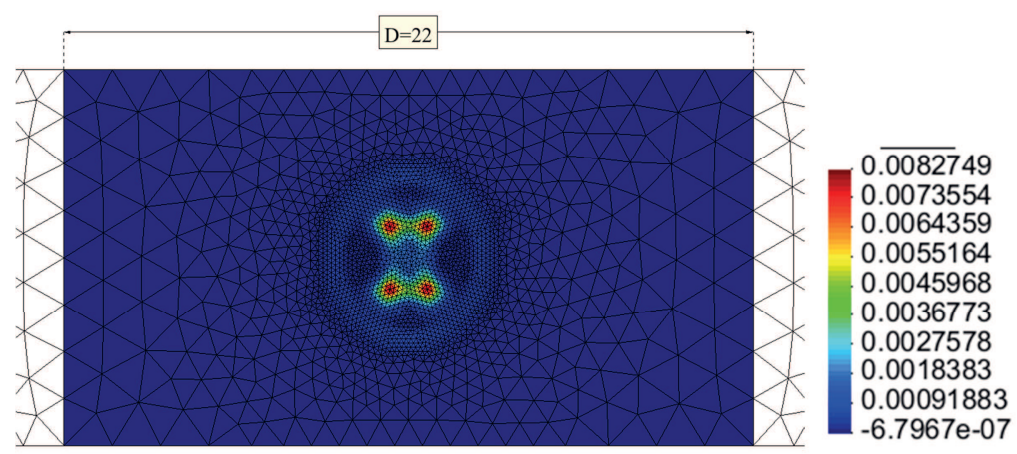

Fig. 13. Cumulative plastic multiplier $\gamma[-]$

Pollack Periodica 11, 2016, 3 


\section{Acknowledgements}

This paper was supported by project SGS15/031/OHK1/1T/11 - Advanced numerical modeling in mechanics of structures and materials.

\section{References}

[1] Gaedicke C., Roesler J., Evangelista F. Jr., Three-dimensional cohesive crack model prediction of the flexural capacity of concrete slabs on soil, Engineering Fracture Mechanics, Vol. 94, 2012, pp. 1-12.

[2] Teixeira M. D. E., Barros J. A. O., Cunha V. M. C. F., Moraes-Neto B. N., VenturaGouveia A. Numerical simulation of the punching shear behavior of self-compacting fiber reinforced flat slabs, Construction and Building Materials, Vol. 74, 2015, pp. 25-36.

[3] Hu H. T., Schnobrich W. C. Nonlinear finite element analysis of reinforced concrete plates and shells under monotonic loading, Computers \& Structures, Vol. 38, No. 5-6, 1991, pp. 637-651.

[4] SIFEL Home page, http://ksm.fsv.cvut.cz/ sifel/, (last visited 20 December 2015).

[5] Krejčí T., Koudelka T., Kruis J. Modeling of building constructions in SIFEL environment, Czech Technical University in Prague, 1. ed., Prague, 2011.

[6] Kruis, J., Matouš, K., Dostál, Z. Solving Laminated Plates by Domain Decomposition. Advances in Engineering Software, 2002, Vol. 33, No. 33, pp. 445-452.

[7] Krejčí, T., Koudelka, T., Kruis, J. Numerical modeling of coupled hydro-thermomechanical behavior of concrete structures. Pollack Periodica, Vol. 10, No. 1, 2015, pp. 19-30.

[8] Kruis J., Koudelka T., Krejčí T. Salt transport in porous materials and its efficient computer implementation, Pollack Periodica, Vol. 9, No. 2, 2014, pp. 111-122.

[9] http://www.code-aster.org/V2/doc/default/en/man_r/r7/r7.01.03.pdf, (last visited 20 December 2015).

[10] Bittnar Z., Šejnoha J. Numerical methods in structural mechanics, ASCE Press, New York, Thomas Telford, co-publisher, London, 1996.

[11] de Souza Neto E. A., Perić D., Owen D. R. J. Computational methods for plasticity, Theory and applications, Wiley, 2008.

[12] Kruis J., Bittnar Z. Reinforcement-matrix modeled by FETI method, Lecture Notes in Computational Science and Engineering, vol. 60, 2008, pp. 567-573. 